\title{
Editorial
}

\section{Applied Mathematics in Biomedical Sciences and Engineering}

\author{
Chang-Hwan Im, ${ }^{1}$ Kiwoon Kwon, ${ }^{2}$ \\ Venky Krishnan, ${ }^{3}$ and Pedro Serranho ${ }^{4}$ \\ ${ }^{1}$ Department of Biomedical Engineering, Hanyang University, 222 Wangsimni-ro, Seongdong-gu, \\ Seoul 133-791, Republic of Korea \\ 2 Department of Mathematics, Dongguk University, Seoul 100-715, Republic of Korea \\ ${ }^{3}$ Tata Institute of Fundamental Research, Centre for Applicable Mathematics, Bangalore 560065, India \\ ${ }^{4}$ Department of Science and Technology, Open University, Campus TagusPark, Oeiras, Portugal \\ Correspondence should be addressed to Chang-Hwan Im, ich@hanyang.ac.kr
}

Received 26 March 2012; Accepted 26 March 2012

Copyright (C) 2012 Chang-Hwan Im et al. This is an open access article distributed under the Creative Commons Attribution License, which permits unrestricted use, distribution, and reproduction in any medium, provided the original work is properly cited.

Biomedical sciences and engineering are representative multidisciplinary research areas that have fulfilled the critical needs of modern medicine and biology. There is no doubt that applied mathematics has played a key role in developing new technologies in these emerging disciplines. For example, modern medical imaging systems such as magnetic resonance imaging (MRI) and computed tomography (CT) could not be materialized without the aid of advanced optimization theories and reconstruction algorithms. Progresses in numerical analysis and biostatistics have also contributed to the rapid advancement of physiological signal processing and computer-aided diagnosis of intractable diseases. Indeed, biomedical sciences and engineering have already become one of the most promising application areas of applied mathematics.

Considering the above-mentioned trends, it seems natural that this journal selected biomedical sciences and engineering as the theme of its Special Issue. This special issue includes fourteen high-quality peer-reviewed articles that might provide researchers in the field of applied mathematics with the current state-of-the-art knowledge of this emerging interdisciplinary research field.

In the paper "Qualitative and computational analysis of a mathematical model for tumorimmune interactions" by F. Rihan et al., a model of differential equations is provided for elucidating the dynamics of tumor growth and immunotherapy interactions, which can predict tumor dormancy and estimate the critical tumor-growth rate. 
The paper "Monitoring personalized trait using oscillometric arterial blood pressure measurements" by Y. Shin suggests a new approach for monitoring personalized trait in oscillometric arterial blood pressure measurements. The proposed approach could offer more reliable blood pressure patterns than the broadly used principal component analysis.

The paper "A linear transformation approach to estimate pulse arrival time" by D. Kim et al. addresses a new mathematical framework for pulse arrival time estimation, combining local characteristics point methods with global parametric methods. The proposed linear method proved to be robust to noise as compared with standard approaches.

In the paper "Modeling of brain shift phenomenon for different craniotomies and solid models" by A. Valencia et al., the authors investigate mathematical models to compute brain displacement caused by craniotomy. The authors predict the displacements and stress levels for three models-elastic model, hyperelastic Ogden model, and hyperelastic Mooney-Rivlin model.

In the paper, "Dynamical models for infectious diseases with varying population size and vaccinations", the authors, P. Shi and L. Dong, propose models for the spread of infectious diseases and vaccinations. They establish results for the existence and global stability of disease-free and endemic equilibria for two cases: susceptible populations are (a) vaccinated continuously and $(b)$ vaccinated once per time period.

In the paper, "Scanning reduction strategy in MEG/EEG beamformer source imaging" by J. Hong and S. Jun, an efficient source scanning strategy in magnetoencephalography (MEG) and electroencephalography (EEG) beamformer imaging is proposed, leading to reduction in the number of scanning points while maintaining good spatial resolution. The efficacy of the new method is demonstrated through numerical and empirical experiments.

In the paper, "Selecting negative samples for PPI prediction using hierarchical clustering methodology" by J. Urquiza et al., a new protein-protein interaction (PPI) support vector machine (SVM) predictor model is proposed. The model is based on using a clustering approach to select a suitable negative data set. The new model is able to classify PPIs under various cases containing positive and negative datasets.

In the paper, "A new weighted correlation coefficient method to evaluate reconstructed brain electrical sources" by J. Choi et al., a novel evaluation metric to evaluate the accuracy of reconstructed MEG and EEG cortical sources is proposed. The new metric reflects the geometry of the cortical surface more accurately.

The paper "Phase- and GVF-based level set segmentation of ultrasonic breast tumors" by L. Gao et al. deals with the segmentation of ultrasonic breast tumors by combining a phase asymmetry approach and a new edge stopping function. They developed a method that can robustly cope with noise and extract the low contrast and/or concave boundaries with high accuracy.

The paper "Mathematical Issues in the Inference of Causal Interactions among Multichannel Neural Signals" by Y. Jung et al. is a well-organized review paper in which the authors described the current state-of-the-art technologies used for the causality inference in the field of neuroscience. It is expected that readers of this journal would be interested in the review paper, since the paper introduces a new interdisciplinary research topic to which their mathematical theories can be applied.

The paper "The second-order born approximation in diffuse optical tomography" by K. Kwon proposes a new numerical method based on the second-order Born approximation for better finding the solutions of diffuse optical tomography (DOT) reconstruction problems. Numerical implementation of the suggested method verifies that the new method has better convergence order than the conventional linearized method. 
In the paper "General computational model for human musculoskeletal system of spine" by K. Kim et al., a general computational model of the human lumbar spine and trunk muscles including optimization formulations is provided. It is expected that the presented computational model and optimization technology can be fundamental tools to understand the control principle of human trunk muscles.

The paper "Personal identification based on vectorcardiogram derived from limb leads electrocardiogram" by J. Lee et al. propose a new method for personal identification using the derived vectorcardiogram (dVCG) from the limb leads electrocardiogram (ECG). The presented experimental results show that it is possible to identify a person by features extracted from a dVCG derived from limb leads only.

In the paper "Coupling of point collocation meshfree method and FEM for EEG forward solver" by C. Lee et al., MEG and EEG forward problems are solved using a new coupling method combining finite element method (FEM) and a point collocation meshfree method. Simulation results show that the hybrid method can be used for efficient computation of EEG and MEG forward problems.

\section{Acknowledgments}

In closing this editorial, we would like to express our deepest gratitude to many reviewers, whose professional comments guaranteed the high quality of the selected papers. In addition, we would also like to express our appreciation to editorial board members of this journal, for their help and support throughout the preparation of this special issue. We hope you will find this special issue helpful for your future study.

Chang-Hwan Im

Kiwoon Kwon

Venky Krishnan

Pedro Serranho 


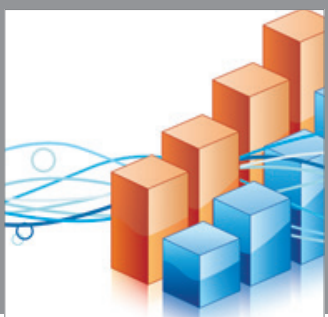

Advances in

Operations Research

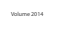

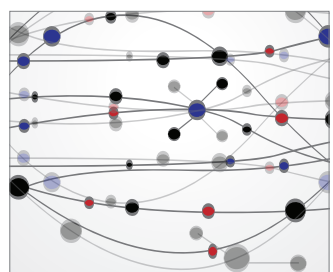

\section{The Scientific} World Journal
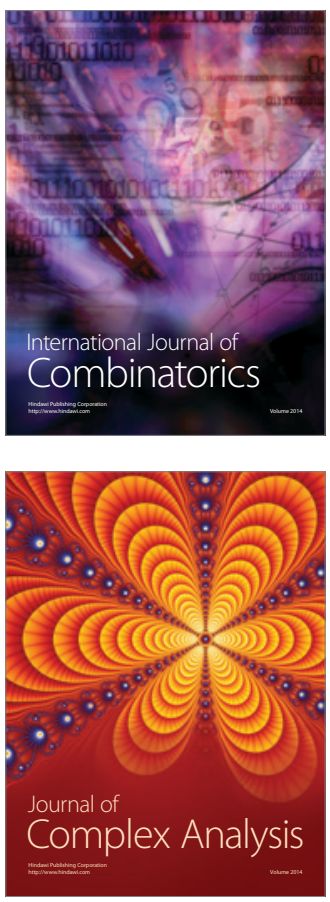

International Journal of

Mathematics and

Mathematical

Sciences
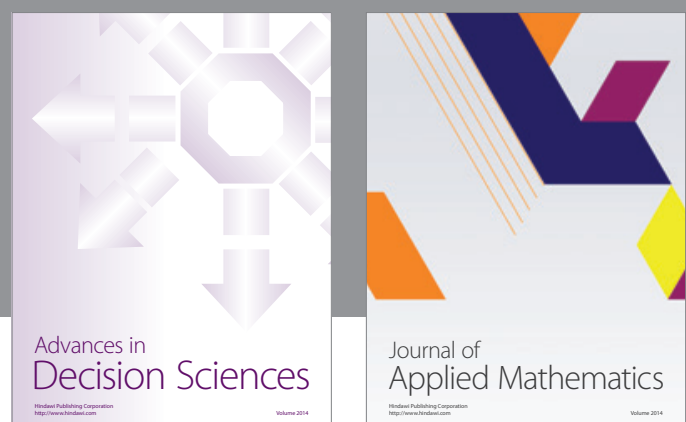

Journal of

Applied Mathematics
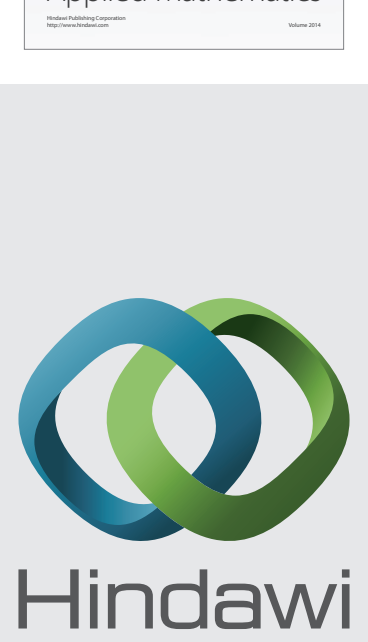

Submit your manuscripts at http://www.hindawi.com
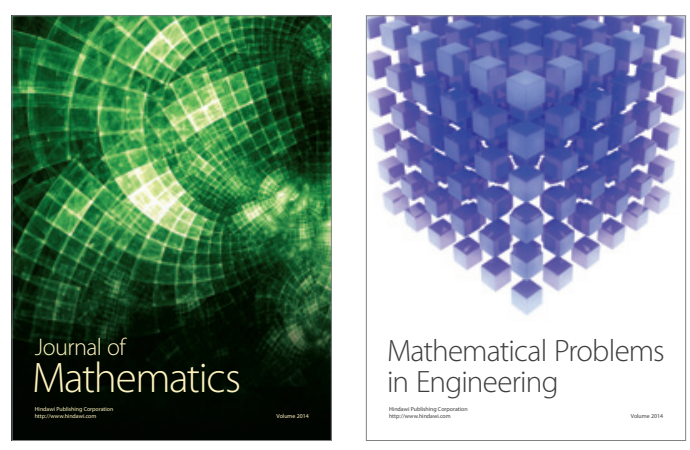

Mathematical Problems in Engineering
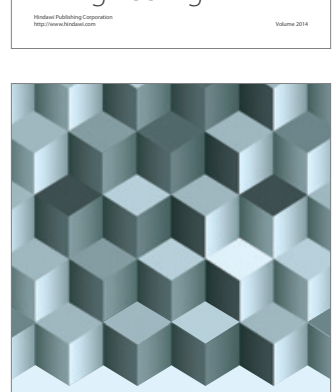

Journal of

Function Spaces
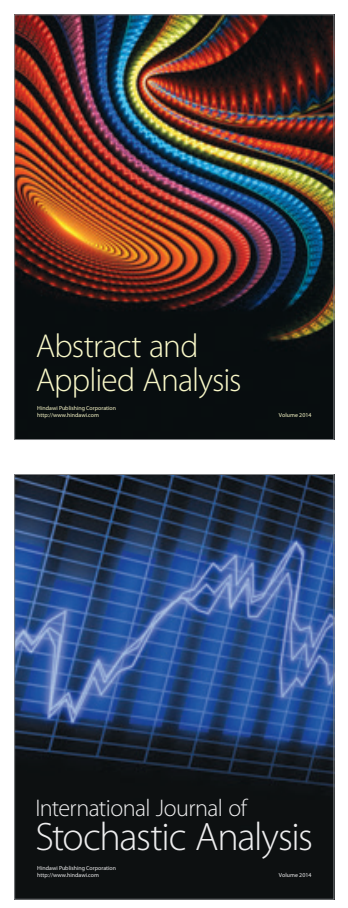

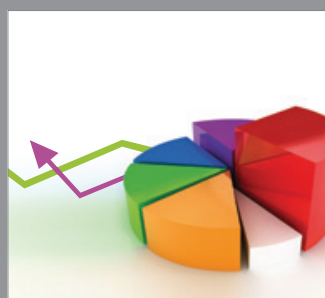

ournal of

Probability and Statistics

Promensencen
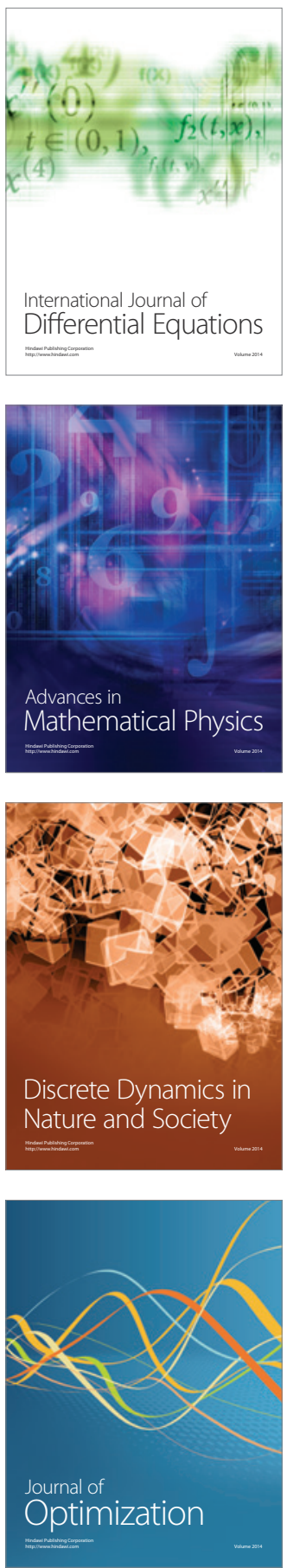